\title{
FarMyo: A Serious Game for Hand and Wrist Rehabilitation Using a Low-Cost Electromyography Device
}

\author{
Thiago Vinícius Vieira Batista ${ }^{1}$, Liliane dos Santos Machado ${ }^{1}$, Ana Maria Gondim \\ Valença $^{2}$, Ronei Marcos de Moraes ${ }^{1}$ \\ ${ }^{1}$ Laboratory of Technologies for Virtual Teaching and Statistics (LabTEVE), Universidade \\ Federal da Paraíba (UFPB), Brazil, \\ thiagovvb@gmail.com,liliane@di.ufpb.br,ronei@de.ufpb.br \\ ${ }^{2}$ Department of Clinic and Social Odontology (DCOS), Universidade Federal da Paraíba \\ (UFPB), Brazil, \\ anamvalenca@gmail.com
}

\begin{abstract}
One of the strategies used in recent years to increase the commitment and motivation of patients undergoing rehabilitation is the use of graphical systems, such as virtual environments and serious games. In addition to contributing to the motivation, these systems can simulate real life activities and provide means to measure and assess user performance. The use of natural interaction devices, originally conceived for the game market, has allowed the development of low cost and minimally invasive rehabilitation systems. With the advent of natural interaction devices based on electromyography, the user's electromyographic data can also be used to build these systems. This paper shows the development of a serious game focused on aiding the rehabilitation process of patients with hand motor problems, targeting to solve problems related to cost, adaptability and patient motivation in this type of application. The game uses an electromyography device to recognize the gestures being performed by the user. A gesture recognition system was developed to detect new gestures, complementing the device's own recognition system, which is responsible for interpreting the signals. An initial evaluation of the game was conducted with professional physiotherapists.
\end{abstract}

Keywords: Serious Game, Gesture Recognition, Virtual Interaction, Rehabilitation, Electromyography;

\section{Introduction}

The use of serious game is not novelty. Over the last years, they have been applied to distinct areas, such as medicine, military and entrepreneurship. Serious games do not have entertainment as their primary goal, but specific tasks such as training, education, advertisement or simulation. One of the areas in which serious games have been applied successfully is in the aid of patients undergoing rehabilitation after a debilitating condition, such as a stroke.

Strokes are a worldwide health issue [1]. They are caused by a disruption of oxygen supply to the brain, usually resulting from rupture of an artery or obstruction by clots, resulting in damage to brain tissue. Although it is not necessarily a fatal condition, strokes cause severe sequelae to the patient and are one of the most disabling conditions [2].

After a stroke occurs, rehabilitation should be started immediately. It is a dynamic process that aims to reduce the resulting disabilities, which can be physical, intellectual and psychological. 
This process occurs through interventions performed by professionals and may involve family and friends [3]. Rehabilitation is performed in clinics and can, sometimes, take place at the patient's house. The process is accompanied by a team of professionals whose goal is to assist patients in all aspects of their life. Physicians, nurses, physiotherapists, speech therapists, occupational therapists, psychologists and social workers usually make up the team that assists these people.

Rehabilitation can be a long-term process. Although crucial to the patient's recovery process, rehabilitation sessions are often discontinued for several reasons. The main causes for interruption are death, stroke complications (stroke recurrence, falls, infectious diseases) and withdrawal due to dissatisfaction with the method or its results [4]. In addition, a possible cause for discontinuation of the treatment for some patients is the difficulty of getting to the clinic, either due to the traveling cost or incompatibility with the clinic's schedule. However, recent studies [5] showed that even when performed at home, patients have difficulty performing rehabilitation exercises. Among the causes of the abandonment is the fact that the patients found the exercises too boring $(25 \%)$ or could not remember how to perform them (19\%). They also declared that motivating and fun games that showed how to perform the gestures with live feedback would help them to continue the treatment.

In recent years, an approach that has been used to enhance commitment and motivation of patients undergoing rehabilitation is the use of computer environments, such as games [6]. They contribute to the rehabilitation process by reducing the monotony of performing several repetitive movements, which is a characteristic of motor rehabilitation, by introducing the electronic games' competitive and playful nature in the treatment, which motivates the user to continue the rehabilitation process. However, there are some problems with these games:

1. Cost: Games for rehabilitation usually involve the incorporation of tracking devices, used by the player to interact with the game and used by the game to acquire data and provide feedback to the player. Some of these devices have high costs, such as robotic equipment, and some are more affordable, like the Kinect. High-cost devices are often inviable for most clinics, so it is important to find low-cost alternatives [7, 8]. In this sense, applications have been developed to investigate how low-cost devices can be used in these games [9] and verify if serious games are positively received by patients [9, 10]. It is important to highlight, however, that the focus of these applications is on technological aspects, such as gesture recognition [9] or on new non-commercial devices [10].

2. Patient Motivation: Making the rehabilitation more inviting and interesting is the reason to introduce serious games in this process, but keeping the patient interested in the rehabilitation game is a challenge. An underwhelming game that fails to capture the patient's attention becomes an experience that is as tiresome as the original treatment. There are many strategies that can be applied in a game to ensure that the patient is motivated, like reward systems or self-adaptive challenges, balanced according to the player's skill level [11].

3. Adaptability: Games should be adapted to the player, especially when dealing with patients that suffered a stroke or some debilitating condition, since they are already under stress and frustration. Therefore, the game should adapt itself to be not too easy, which would be boring for the player, nor too hard, which would frustrate the patient even more $[12,13]$. For example, a dynamic difficulty adaptation technique can be used [13], which differs from the commonly used techniques of incremental difficulty adaptation and random difficulty adaptation. This technique is based on the generation of game levels adapted to each patient. It has been tested on an upper limb rehabilitation game and the results indicated that this strategy had positive impacts on the rehabilitation session, both in the treatment and in the patient's motivation. 
In this work, a serious game for supporting hand and wrist rehabilitation is presented, bringing a low-cost system capable of improving patient commitment to the treatment and that adapts itself to the skill level of the patient. The game requires that the user executes all gestures involved in the rehabilitation of hand and wrist to accomplish its established goals, making it a viable tool to be used in clinics.

\section{Methodology}

The development of the serious game consisted in three main steps: game concept, gesture recognition and content tests. The development team included professionals from several areas, with emphasis on a physiotherapist, who provided support during all phases of the development process.

\subsection{Game Concept}

One characteristic that is commonly found in rehabilitation games is that they focus mostly on technical aspects, while the game itself (e.g. story and characters) is not given the same amount of attention. Schell [14] defined the four elements that compose a game, organizing them in the game's elemental tetrad: Aesthetics, Story, Mechanics and Technology. This tetrad was further expanded in the context of serious games [15], with another element added: the specific content that the games of this nature are built for, which directly affects the conception of every element of the game (Fig. 1).

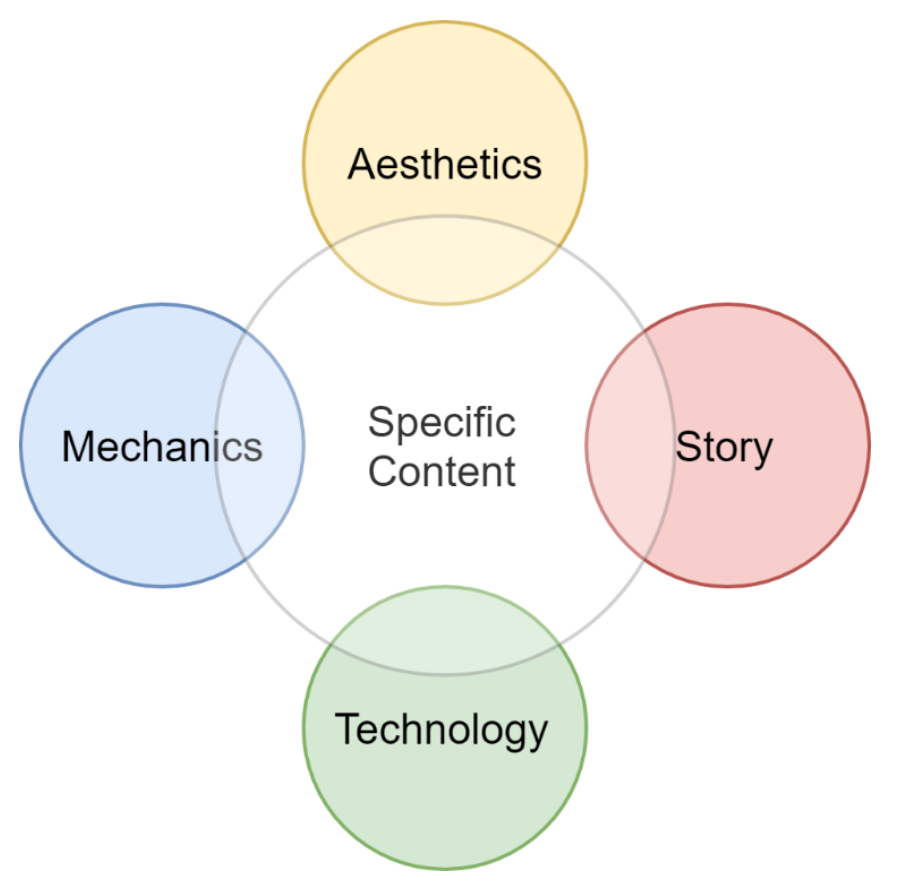

Figure 1. Expanded Schell's Elemental Tetrad [15]

According to this expanded tetrad, a serious game must integrate the specific content to the previous four elements, affecting the way the story is set, fitting the technology to the game's purpose, balancing the mechanics to user's skills and presenting aesthetic elements that support, without distracting, the user, in the sense of achieving the desired goals. Since the purpose of using a rehabilitation game is keeping the patient interested, the game was designed to consider these aspects, otherwise they would lose interest and the game would lose its purpose. 
pag. 6

\subsubsection{Requirements}

The target group of the game are patients in the process of hand and wrist rehabilitation. The design focused on the use of the game during rehabilitation sessions in clinics, with the assistance of a physiotherapist. Thus, the specific content of the game must be based on a set of exercises known by rehabilitation professionals. A physiotherapist was integrated into the project, providing information regarding the gestures necessary in a system for hand and wrist rehabilitation (Fig. 2). They also indicated that elbow exercises are important in this kind of rehabilitation process, as illustrated in Fig. 3.

Wrist Exercises

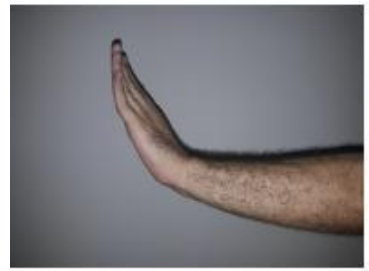

Extension

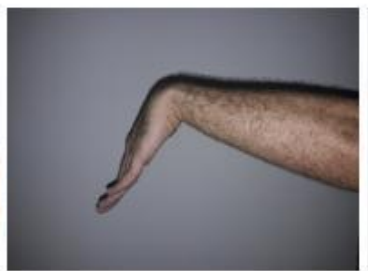

Flexion

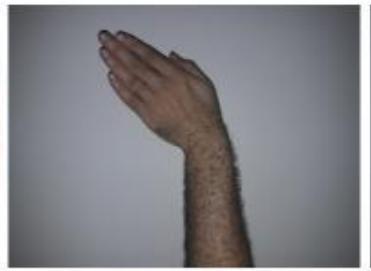

Ulnar

Deviation

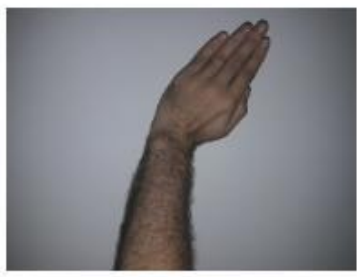

Radial

Deviation

Hand Exercises

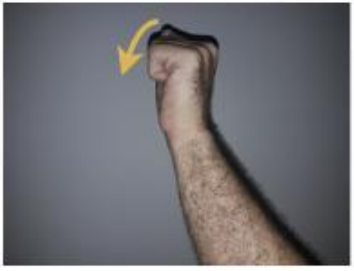

Flexion

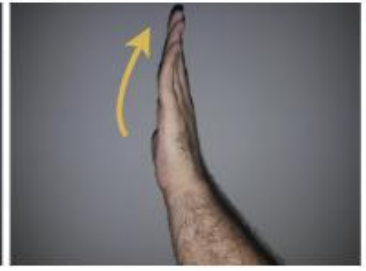

Extension

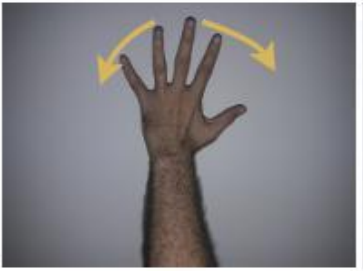

Abduction

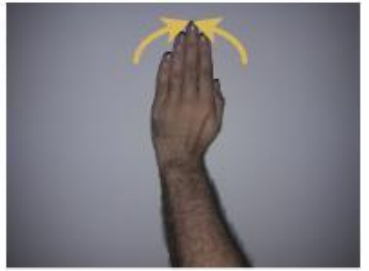

Adduction

Figure 2. Hand and wrist gestures

\section{Elbow Exercises}

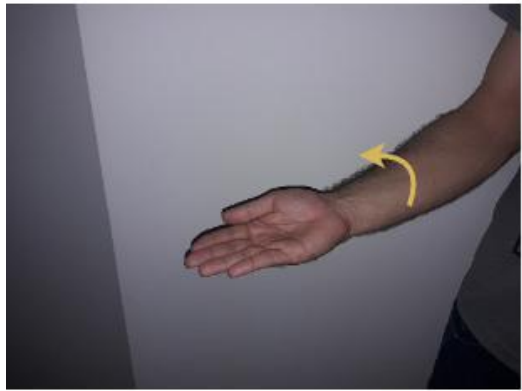

Supination

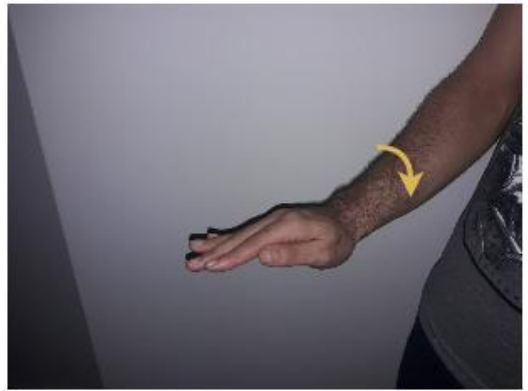

Pronation

Figure 3. Elbow exercises

The physiotherapist also indicated that the game has as target group a specific set of patients, determined with basis on the Medical Research Council (MRC) scale for muscle strength [16]. This scale quantifies the patient's muscle strength in six levels, that are shown in Table 1. 
Proposed in 1943, this scale has been used ever since as a reference in muscle strength. Taking this scale in consideration, it was defined that the game would be mainly indicated for patients in level 3 or 4, since patients with muscle strength below level 3 usually will be unable to perform movements by themselves, without help from the therapist, and patients in level 5 usually don't require rehabilitation.

Table 1. Medical Research Council Scale for Muscle Strength.

\begin{tabular}{c|c}
\hline Type & Description \\
\hline $\mathbf{0}$ & No contraction \\
\hline $\mathbf{1}$ & Only a trace of contraction \\
\hline $\mathbf{2}$ & Active movement, with gravity eliminated \\
\hline $\mathbf{3}$ & Active movement against gravity \\
\hline $\mathbf{4}$ & Active movement against gravity and resistance \\
\hline $\mathbf{5}$ & Normal strength \\
\hline
\end{tabular}

\subsubsection{Device and Technology}

In serious games for rehabilitation, gesture recognition plays an important role. Gesture recognition is a process in which gestures made by the user are recognized by the computer. It can involve motion of the fingers, hands, arms, head, face or body with the intent of interacting with the environment or expressing an information [17] and it is also used to assess user performance and guarantee that the gestures are being executed correctly. Normally, optic devices are used to perform gesture recognition [18]. However, advances in physiological sensing have allowed the use of electromyography in these systems.

Electromyography is a technique that consists in the measurement of the electrical signals produced by the muscles when there is a stimulation of the motor units (MU). These consist of motoneurons (present in the spinal cord), each of which is attached to one or more muscular fibers of the human body [19]. When stimulated, the muscular fibers produce an action potential, named Muscle Fiber Action Potential (MFAP). The sum of the action potentials of all muscular fibers belonging to a MU is named Motor Unit Action Potential, which comprises the electromyographic signal. The fibers' physiology determines the amplitude, shape and the duration of each MFAP, defining the characteristics of the electromyographic signal [20].

The two main techniques for quantifying electromyographic signals are the intramuscular and surface electromyography. In the first, the sensor is inserted in the user's body. Although more precise than the other alternative, it has the disadvantage of being a potentially painful and invasive technique. Surface electromyography is a safer and non-invasive technique, because it captures the signals generated in the MU through sensors positioned over the user's skin.

For the serious game, the Myo Armband was used, a low-cost surface electromyography commercial device that provides raw data when reading user movements. Previous work [21] showed that the Myo has good accuracy for classifying electromyographic signals for hand prosthetics. A setup consisting of both a pair and a single Myo was tested against other setups, with the Myo obtaining good results, similar to those obtained by much more expensive devices. Kerber, Puhl and Kruger [22] also developed a system for recognizing gestures using the Myo armband with an accuracy of 95\%, using a Support Vector Machine as their machine learning algorithm.

Myo is a surface electromyography device that consists of eight sensors positioned over the user's skin, resembling an armband. They collect electromyographic data and use this information to detect gestures made by the user. Myo was initially not intended to be used as a rehabilitation tool, but as an interaction gadget to control devices such as sound players, televisions, tablets and even drones. However, by using the Myo programming library, it is possible to access to the raw electromyographic data. This device has the advantage of being available at an affordable price 
(approximately 250 USD at the time of the writing of this document). Myo was chosen because of its relative low cost and ease of use, which could facilitate the incorporation of this system in clinics. Fig. 4 shows the device in use. Unfortunately, in late 2018, Myo's production and sales have been discontinued. However, there are other alternatives being developed and already available, like LeapMotion [23] and Kai [24], that could be used to replace Myo.
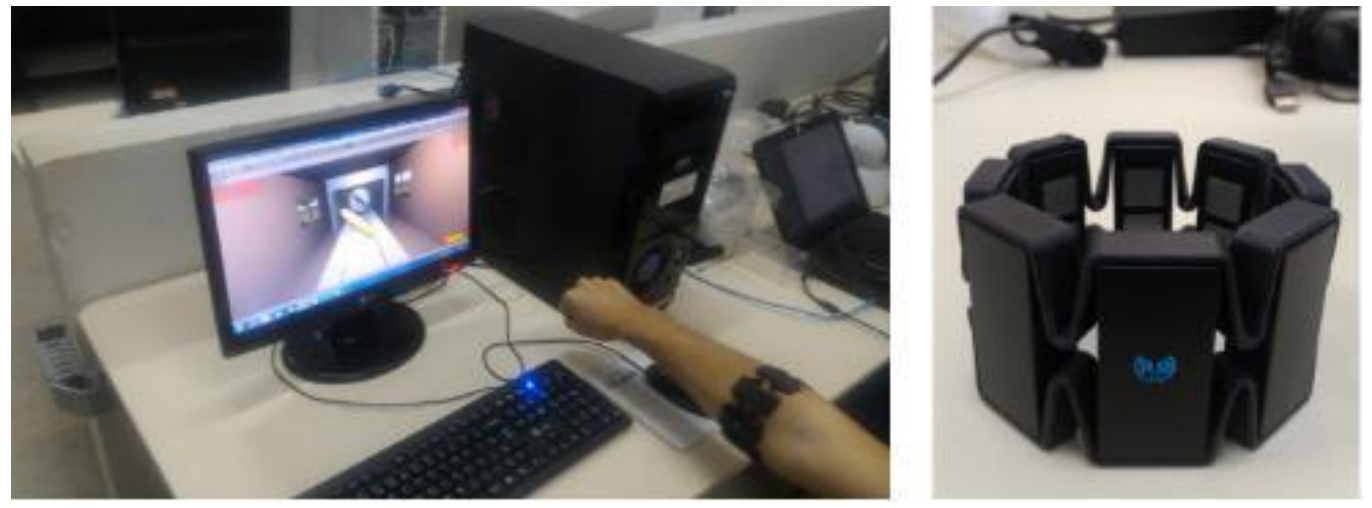

Figure 4. Myo (right) and a player using it to interact with a game (left).

\subsubsection{Motivation and Adaptation}

Studies indicated the importance of guaranteeing a good player experience [25] and that enjoyment is an important aspect in the success of serious games [26]. Thus, strategies can be used to improve user experience in rehabilitation games, increasing users' interest in the treatment and reducing the monotony of performing several movements in a repetitive manner.

The design of the serious game proposed included a system of rewards and specific goals to be achieved, both integrated to its ludic and competitive nature. Furthermore, it is known that the capacity of serious games to provide feedback regarding patient performance enhances the quality of the treatment and motivates them to dedicate a larger amount of time for performing the exercises [27]. Thus, difficulty adjustment is one of the most important aspects of a serious game for rehabilitation, improving patient motivation and engagement [28] because it avoids situations where patients are faced with exceedingly difficult challenges that would frustrate them. In this work, a dynamic difficulty adaptation approach was adopted, with the difficulty being automatically adjusted by the system according to user's actions.

\subsection{Gesture Recognition}

Devices based on surface electromyography can be an alternative to commonly used devices in rehabilitation applications because they are non-invasive, low-cost and present a safe way to capture muscular signals and interpret this information as a gesture [29]. Surface electromyography is a promising approach in rehabilitation applications because it is a noninvasive technique that captures signals through sensors positioned over the user's skin [18]. However, some of the commercially-available devices, which are usually more affordable and accessible, were likely not designed for use in rehabilitation, so they do not recognize all the gestures and movements required for this purpose. Thus, the incorporation of a decision model that can interpret the information collected by the device as gestures or movements may be necessary in order to provide feedback to the patient regarding gesture performance.

The Myo system already recognizes some gestures used in hand exercises, so an additional model to recognize these was not necessary. Additionally, Myo possesses a gyroscope, which is enough to recognize the elbow exercises with the Myo development library. A new gesture 
recognition system is necessary only to address wrist exercises, which are not supported by the Myo system: extension, flexion, ulnar deviation and radial deviation. A summary that clarifies which gestures needed to be recognized by our model and which gestures were handled by the device's system is presented in Table 2 .

Table 2. Summary of the division of the gesture recognition

\begin{tabular}{|c|c|c|}
\hline Region & Gesture & Recognition System \\
\hline \multirow{4}{*}{ Wrist } & Flexion & Own model \\
\cline { 2 - 3 } & Extension & Own model \\
\cline { 2 - 3 } & Ulnar Deviation & Own model \\
\cline { 2 - 3 } & Radial Deviation & Own model \\
\hline \multirow{2}{*}{ Fingers } & Flexion/Extension & Myo \\
\hline \multirow{2}{*}{ Elbow } & Abduction/Adduction & Myo \\
\hline
\end{tabular}

Different models have been used for gesture recognition, such as Bayesian networks, neural networks and fuzzy-logic-based classifiers. Since there is no model which solves all gesture recognition problems, a model must be chosen carefully, since some of them have computationally demanding calculations that would make them unsuitable for real-time applications. In this sense, neural networks are a good choice for solving this problem. Although the network training takes a substantial amount of calculations, after its completion the classification itself is performed almost instantaneously.

Neural networks are structures inspired by the organization of the human brain, composed by a network of neurons that communicate and are capable of learning by analyzing activity patterns, with information stored as weights in the connections between the neurons. A neural network is composed by a set of nodes (representing the neurons) connected by arrows (representing the synapses). One of the types of neural networks is the Multilayer Perceptron, which uses a network with a set of layers: an input layer, one or more hidden layers and one output layer. Networks of this kind have been used successfully to solve different problems by applying the backpropagation learning algorithm [30] to train the network.

In this work, a neural network was used to classify the electromyographic data. The neural network was chosen because it achieved the best results in the tests performed, which were described in Batista et al. [31].

\subsection{Tests}

Two different test sessions were conducted in this work. The first one took place when the prototype of the game was developed. This test was intended to verify the adequacy of the device to the target group and the acceptance of the application by a patient. This test was a limited empirical study with a single potential user.

The second test session was performed for evaluating the final game. A group of three professionals was invited to play the game and describe their impressions regarding the game adequacy for rehabilitation of hand and wrist, system usability, game importance, viability of use and their opinion about the device used. The interviews were recorded with their permission. The profiles of the three professionals are:

Professional 1: A physiotherapy professor with experience in clinical rehabilitation.

Professional 2: A university physiotherapy professor.

Professional 3: A physiotherapy professional with experience in clinical rehabilitation. 


\section{Results}

With all the components of the game and its overall organization defined, this section describes the results of each step in the development process. Later, a discussion about the considerations of professionals of the area after playing the game and their opinions on its benefits, viability and usefulness is presented.

Taking the modified Schell's elemental tetrad [15] into consideration, the game "FarMyo" was created to aid patients undergoing hand and wrist rehabilitation. The game consists of an environment where the user can select one of three mini-games, each of them treating a different group of gestures. Aesthetically, the game is farm-themed, with an idyllic farmland scenery composed of structures that resemble a barn, a vegetable garden and a henhouse. Graphical realism was not one of the priorities for the scenario of the game. The focus was on clear and simple elements that would ease the identification of elements of the scenario and of the activities to be performed. Story-wise, the game consists of the story of a farm owner, who produces different items and sells them. The user must help the owner collect or process the items in each mini-game to meet a daily quota. The game engine Unity3D and the Myo SDK were used to develop the game.

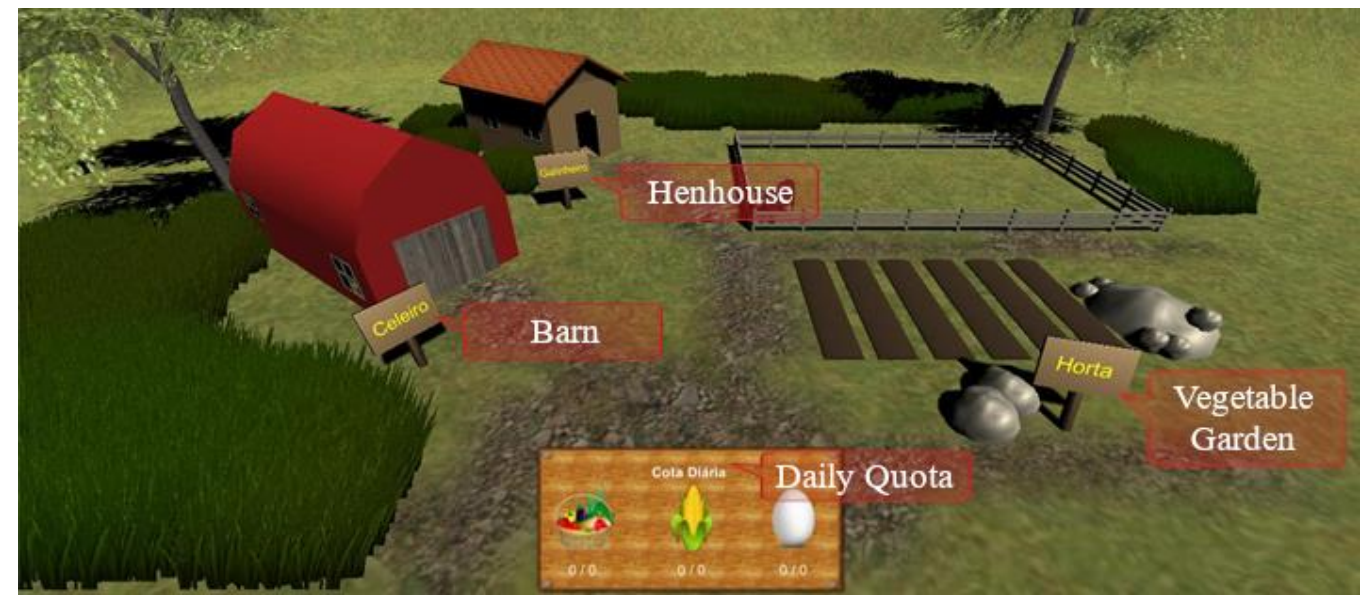

Figure 5. FarMyo's environment.

Fig. 5 shows the game environment, which consists of three main structures representing the three available mini-games. Each of them addresses a different set of rehabilitation exercises. To complete the game, users must collect all the items from each mini-game.

Each gesture was integrated into the story with help of a professional, who indicated the best way to adapt the rehabilitation movements to the game:

1. The barn: illustrated by the red structure in Fig. 5. It represents the game of corn collecting, which deals with elbow exercises that are important in the process of hand and wrist rehabilitation.

2. The henhouse: illustrated by the beige-colored structure in Fig. 5. It contains the eggcollecting game, which deals with exercises involving the fingers.

3. The vegetable garden: illustrated by the brown-stripped structure in Fig. 5. It represents the fruit collection game, which deals with wrist exercises.

The barn mini-game consists of a conveyor belt and corn cobs that must pass through a grinder and be collected, as illustrated in Fig. 6. While the corn moves in the direction of the grinder, the user rotates their arm to the right and to the left, adjusting the corn cob's rotation and by consequence performing the elbow exercises, pronation and supination. The system adjusts 
difficulty automatically, by decreasing the speed that the corn cob moves towards the grinder if the player fails and increasing the speed otherwise.

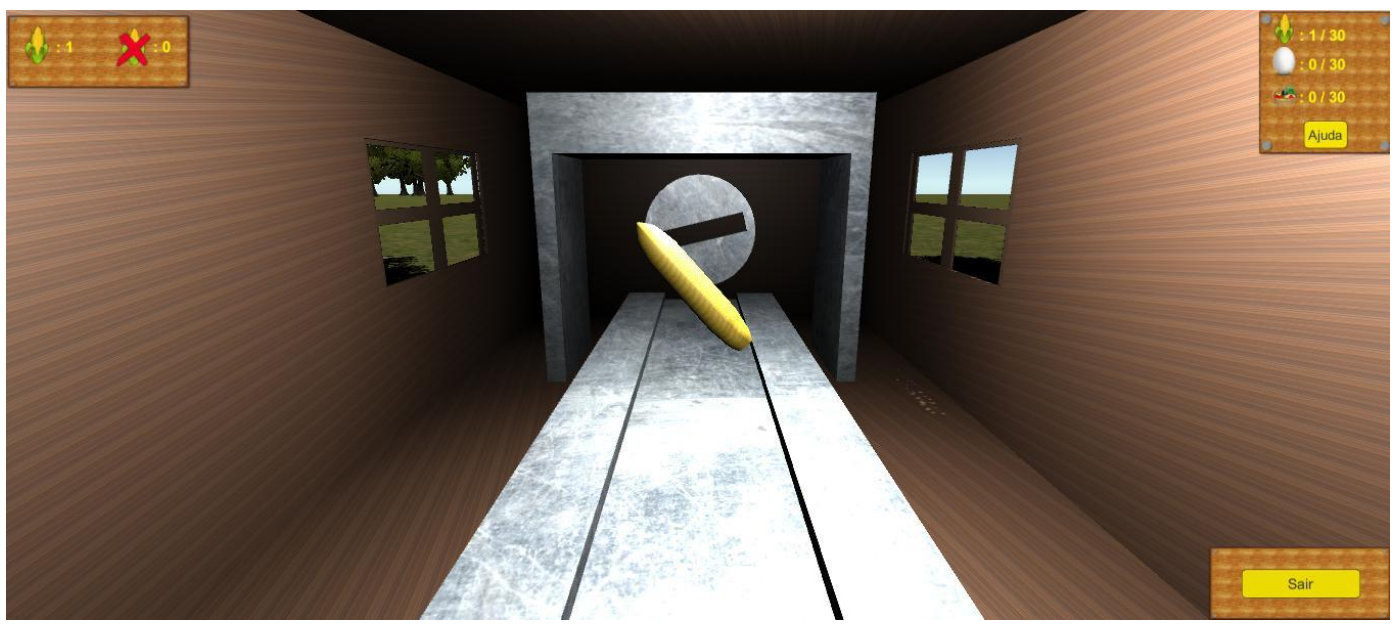

Figure 6. The barn mini-game. The corn cob must be rotated by user's movements to pass through the grinder.

In the henhouse mini-game the player collects eggs that come down rolling, as illustrated by Fig. 7. Players have control over the boxes, and they must allow the good (white) eggs to fall inside the boxes by opening them and prevent the rotten (gray) eggs from falling inside the boxes by closing them. To open and close the boxes, the user must perform the hand exercises: flexion, extension, abduction and adduction. The automatic difficulty adjustment system controls the speed that the eggs roll down, depending on the user's ability to fulfill the objectives.

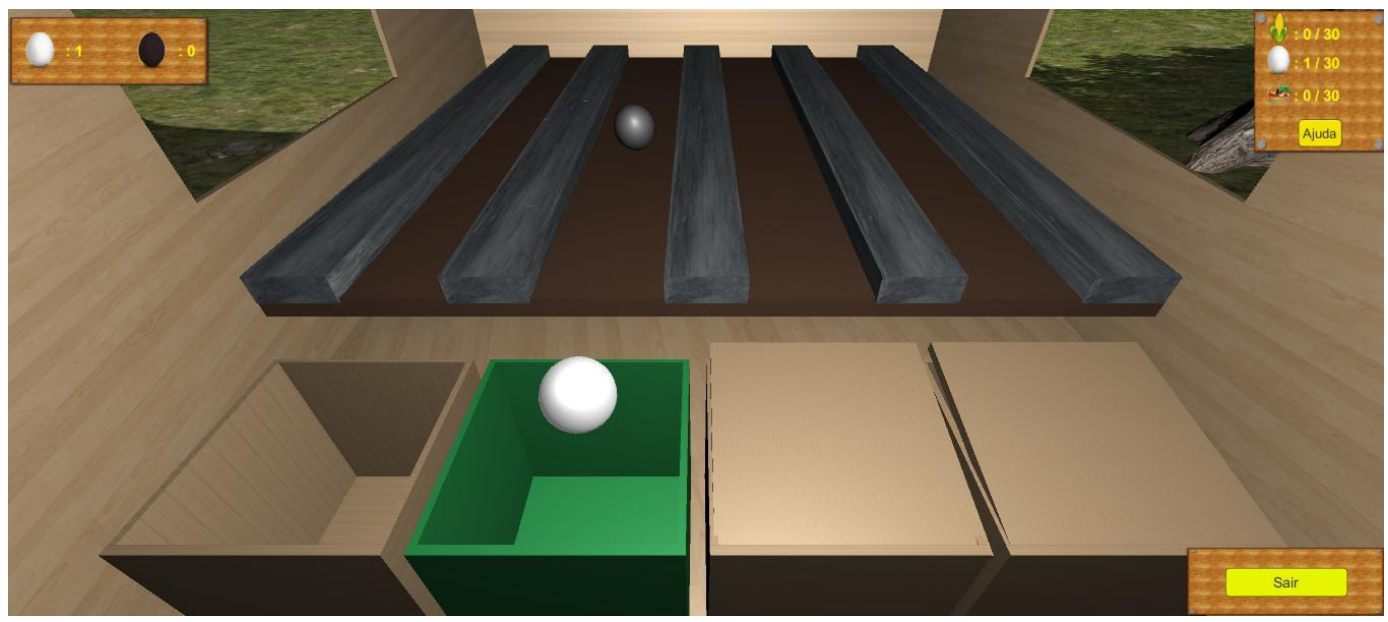

Figure 7. The henhouse mini-game. The green box indicates the box selected by the user to be opened or closed.

The vegetable garden mini-game consists of trails with stones that roll along them. The player must reach the other side and collect fruits while avoiding collision with the stones, as illustrated by Fig. 8. The wrist exercises (extension, flexion, ulnar deviation and radial deviation) must be performed in order to move forward, backward, left and right, respectively. The last two exercises are adjusted depending on which arm is currently being used to play the game. The automatic adjustment system will reduce or increase the speed of stones depending on the player's performance in the game. 


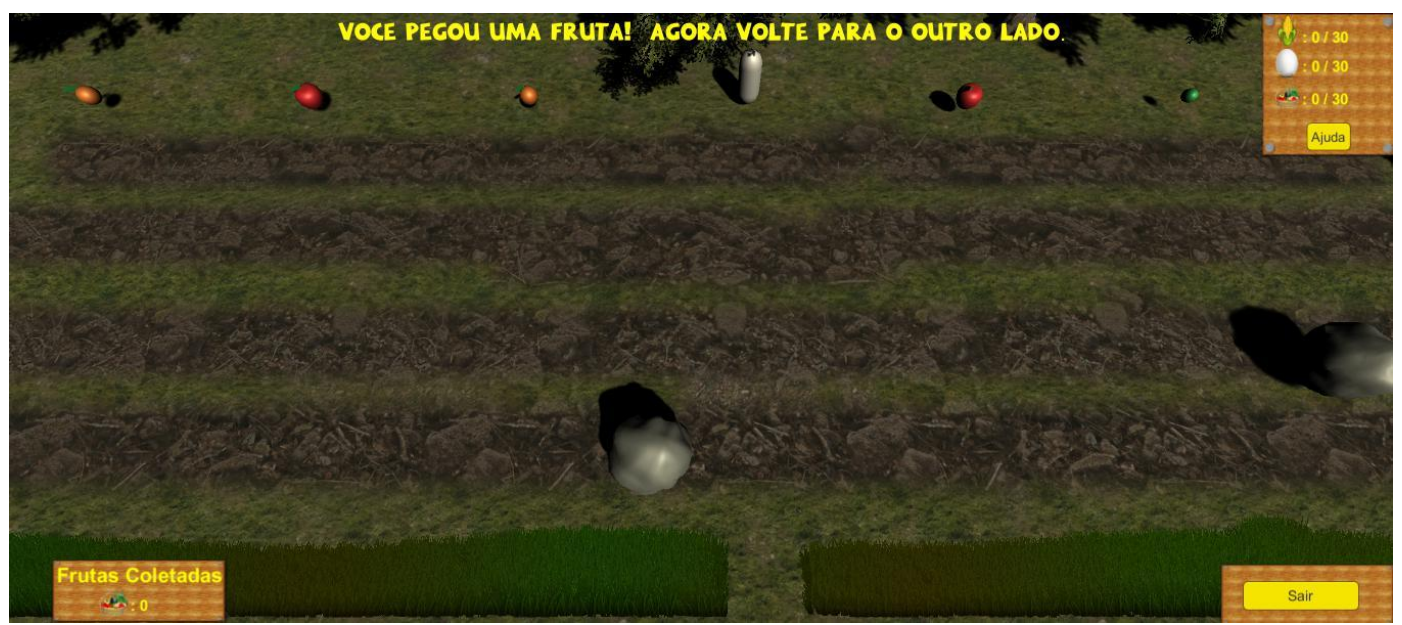

Figure 8. The vegetable garden mini-game. The user's avatar starts on the bottom and must be moved to the top to collect fruits.

\subsection{Gesture Recognition System}

For the gesture recognition, the data is initially collected by the device. Then, the raw data provided passes through a process of feature extraction. The data processing is similar to the one described in Batista et al. [31], but in this case, a different decision model was used. The data is analyzed by the chosen decision model, multilayer perceptron neural network, to recognize the gestures. The architecture of the system is illustrated in Fig. 9.

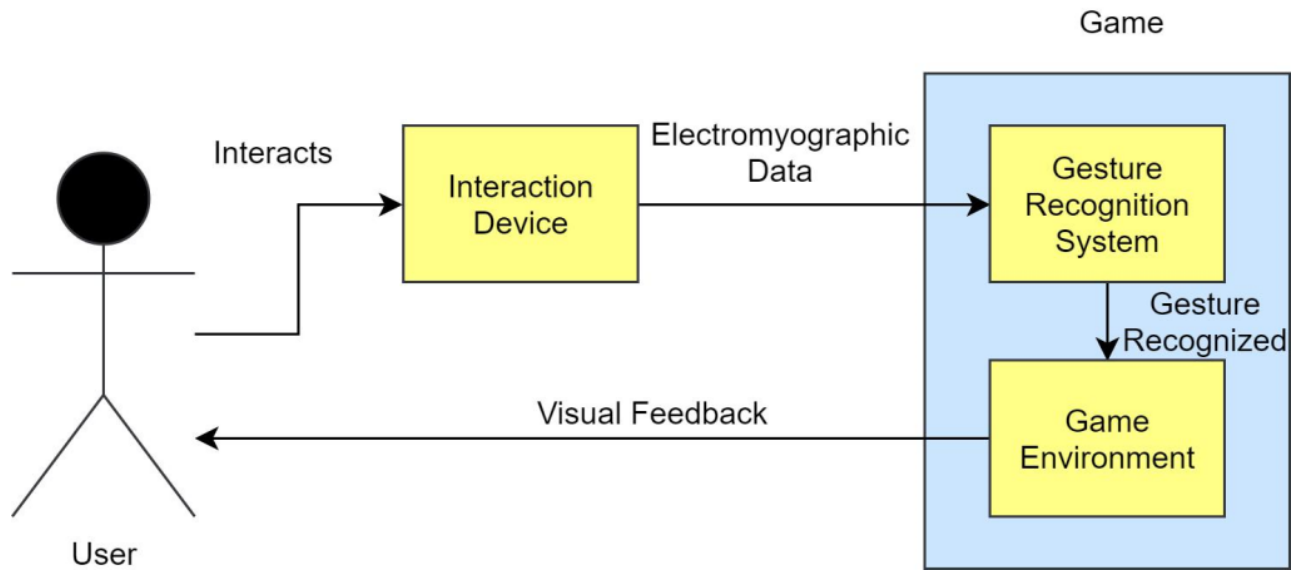

Figure 9. Architecture of the game integrated with the Myo system.

Since the device provides a constant flow of electromyographic data, detecting when a gesture is executed can be difficult because the signal of a gesture is not clearly bound, which means it is not trivial to tell when it begins and ends. Therefore, the strategy of slicing data in half-second intervals of electromyographic data was adopted, and each of the slices is processed as an independent gesture by the algorithm, making up the samples. An example of the signal separation is illustrated in Fig. 10. 


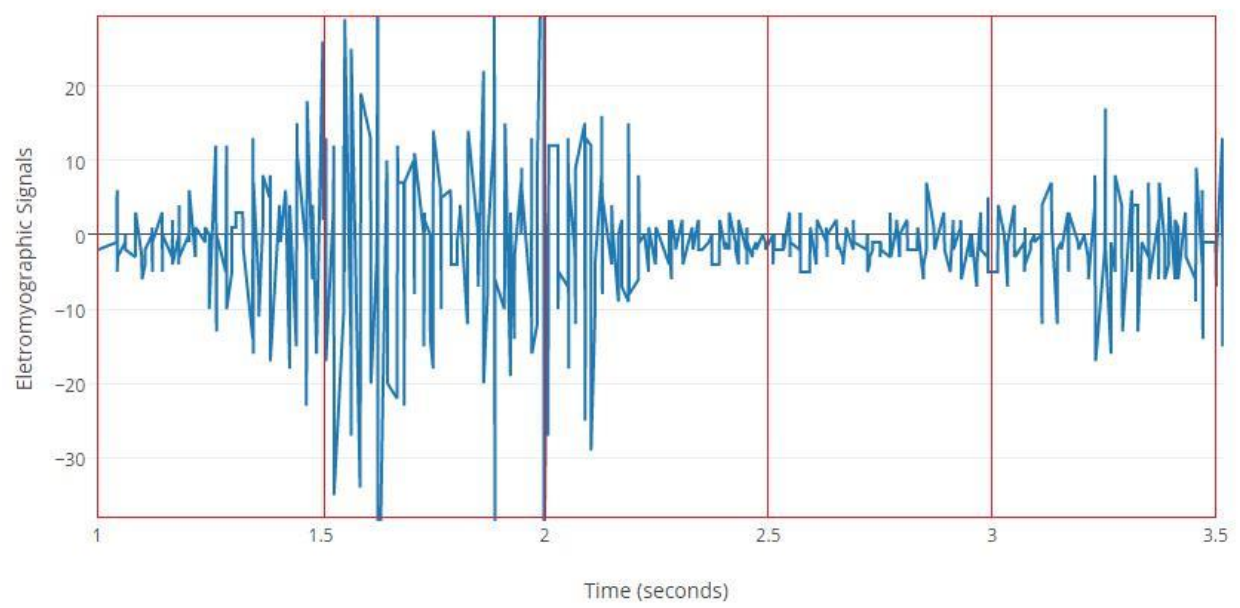

Figure 10. Electromyographic signal separation.

To process the raw data, it was necessary to perform the process of feature extraction, which means transforming the raw data into variables, called features, used to train the learning algorithm [32]. In this case, the raw data are the electromyographic signals captured by the device. A simple method for extracting the features was used: for each piece of signal, only the highest and lowest values are stored. Mathematically, this can be expressed as:

$$
\begin{aligned}
& \hat{x}_{\text {min }}=\min \left(x(i)_{1: t}\right) \\
& \hat{x}_{\max }=\max \left(x(i)_{1: t}\right)
\end{aligned}
$$

where $\hat{x}$ is the feature extracted, $i$ is the sensor from which data was extracted, $x$ is the electromyographic data and $t$ is the length of the dataset. Since there are 8 independent sensors, 16 features will be extracted, representing the highest and the lowest values registered by each sensor in that piece of the signal. These highest and lowest values are represented by the purple dots in Fig. 11.

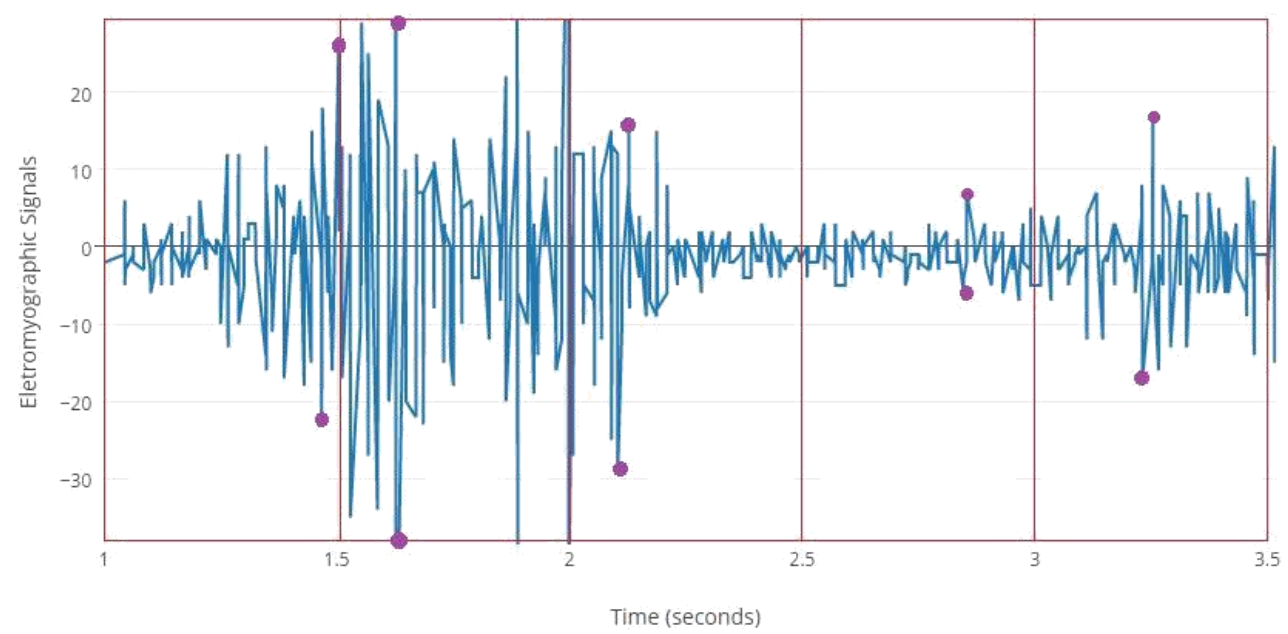

Figure 11. Electromyographic signal separation with the extracted features.

Since the electromyography device does not recognize all the necessary gestures, a new gesture recognition system using Myo raw data was implemented, as shown before. To assess the system's capability of classifying gestures from electromyographic data, an experiment was 
pag. 14

conducted with an user performing the four wrist gestures and also standing in the resting position. For this test, a database with 500 samples was used. Each gesture was represented by 100 samples, split into 70 test samples and 30 training samples, making up 350 test samples and 150 training samples. The measurements were made by a 24 -year-old individual wearing the Myo on the right arm.

The neural network used in this work is shown in Fig. 12. It has 16 input nodes, referring to the 16 features extracted for each gesture, and 5 outputs, which represent the 5 possible classifications: Flexion, Extension, Ulnar Deviation, Radial Deviation and Resting Position. The network parameters were a learning rate of 0.3 and a momentum of 0.2 .

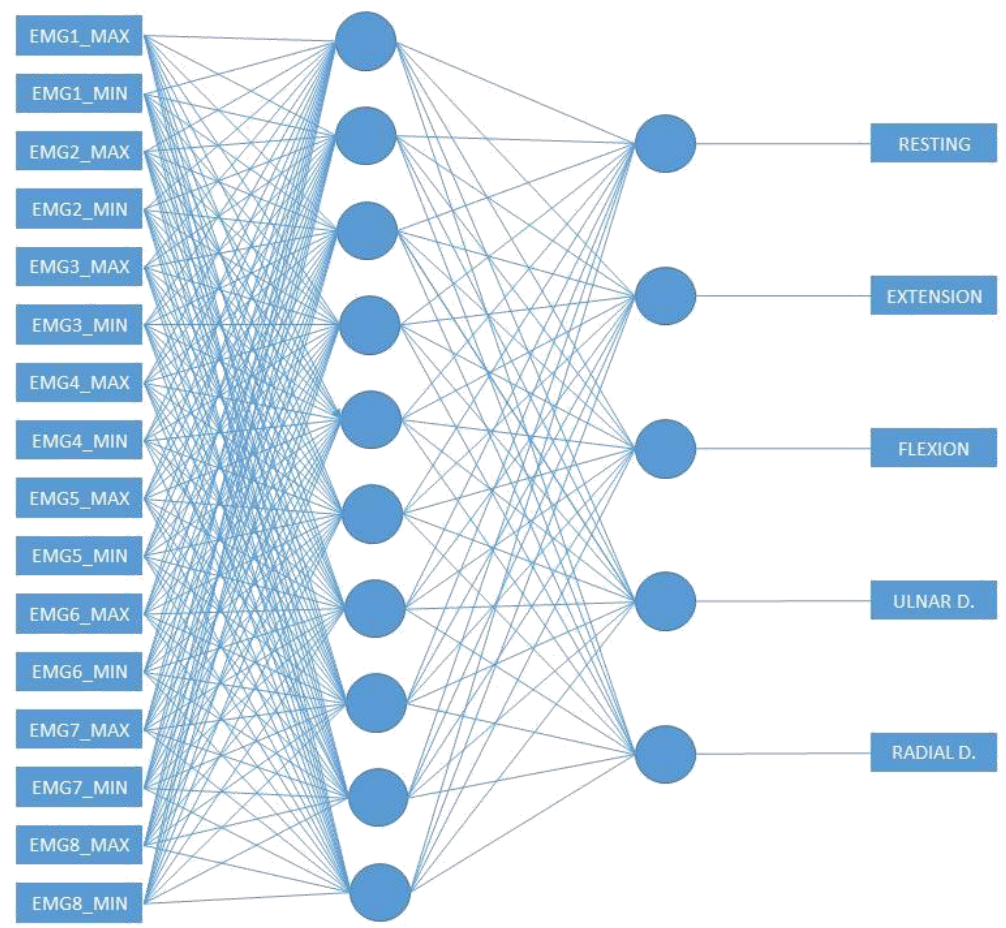

Figure 12. Neural network used in this work.

The results suggest that the system had an acceptable performance, as shown by Table 3, which represents the confusion matrix obtained. The model had an accuracy of approximately 98.3\% when classifying electromyographic data.

Table 3. Confusion matrix for the tests performed with the model.

\begin{tabular}{c|c|c|c|c|c}
\hline & Resting & Extension & Flexion & Ulnar D. & Radial D. \\
\hline Resting & $\mathbf{7 0}$ & 0 & 0 & 0 & 0 \\
\hline Extension & 0 & $\mathbf{7 0}$ & 0 & 0 & 0 \\
\hline Flexion & 0 & 0 & $\mathbf{7 0}$ & 0 & 0 \\
\hline Ulnar D. & 3 & 0 & 1 & $\mathbf{6 6}$ & 0 \\
\hline Radial D. & 0 & 0 & 0 & 2 & $\mathbf{6 8}$ \\
\hline
\end{tabular}

Therefore, the model can be considered adequate for use as an efficient decision model for classifying wrist gestures. 


\subsection{Testing the device}

To assert if the device is viable for patients in the process of rehabilitation, a test was performed with a voluntary individual who was in the process of recovery after a stroke. He was, at the time of the test, in the level 3 of the MRC scale. The test was performed with the assistance of a rehabilitation professional.

In the test, the individual was asked to perform some movements to verify if the device could capture the corresponding signals. Additionally, the calibration routine of the device was performed. In both cases, the test had a positive outcome, as the device could capture the individual's electromyographic data and the calibration routine was successful.

Finally, the patient stated that the device was comfortable and easy to use and that he could barely notice that he was wearing it, since the device weighs only 93 grams. He also stated that he would have no problem using it for a prolonged period of time and that he would be interested in playing a serious game for the purpose of rehabilitation.

\subsection{Automatic Adaptation System}

Aiming to maintain patient interest in the game and implement adaptability, an automatic game balance system was developed, which maintains the game in a level adequate to the patient playing at a given moment. The adjustment is made through a rule-based system [33], based on classic logic. It consists of a knowledge base where the knowledge is represented by a set of rules of the type: if < condition > then < conclusion >.

A rule-based system incorporates rules that describe relevant situations and the corresponding actions [31]. In this game, the rules correspond to the verification of situations of failure or success and the actions are the decrease or increase of the game difficulty in fixed values. For instance, if the player fails to collect a product, the difficulty of the game is slightly decreased (Fig. 13). Consequently, if the player succeeds in collecting a product, the difficulty is increased in the same proportion.

With time, the game will adjust itself to the skill level of the player. If a player's abilities exceed the game's difficulty, for example, this player will probably succeed more, and so the difficulty would be raised until it reached a point of balance between successes and failures. When the session ends, these parameters are stored in the user's profile.

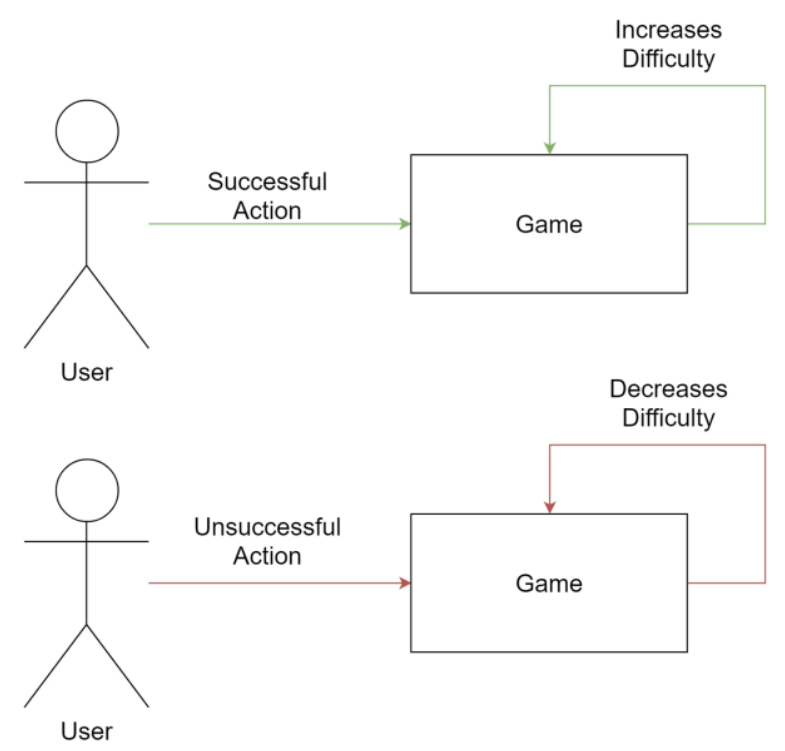

Figure 13. Automatic adjustment system. 


\subsection{Patient Motivation}

To improve patient motivation and engagement, resources to provide feedback to users were implemented: a display of daily quota, available in all scenes and mini-games, and a system of achievements. The daily quota display allows users to see when a correct movement was performed and converted into an item processed or collected. This display can be observed at the bottom of Figure 5 and at the top-right of Figures 6, 7 and 8.

The achievement system rewards patients during the rehabilitation process. With this system, patients are rewarded for completing a certain set of tasks in the game, so they can have a sense of accomplishment when playing the game in rehabilitation sessions. Some elements of the achievement system can be seen in Fig 14.

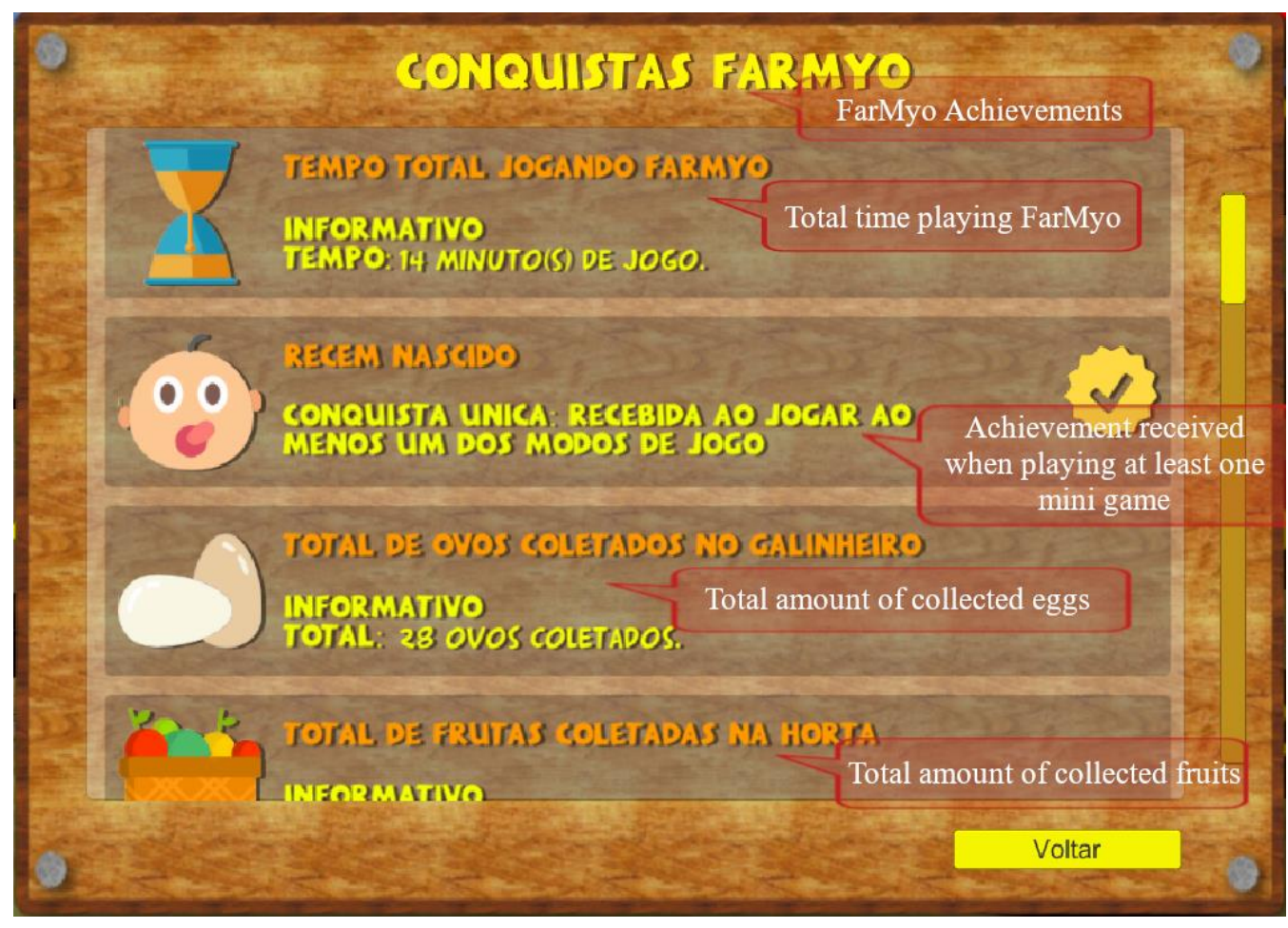

Figure 14. FarMyo achievement screen (in Portuguese)

\subsection{Professional Feedback}

After the conclusion of the game's development, tests were performed with three professionals, as described in section 2.3. Professional 1 stated that the game is important in the process of patient rehabilitation, being an attractive tool that makes the treatment more dynamic. He also indicated that the patients see games as a challenge, wanting to break their limitations and succeed. He thought the device was simple and could be applied to a clinical environment, while stating that the cost would be acceptable for this end. Regarding the gestures incorporated, he stated that the basic movements for hand rehabilitation are present. Finally, the professional indicated that the game has the potential to be used in rehabilitation at home.

Professional 2 enjoyed the game, pointing as main advantages of the game the "dynamization" of the treatment, which motivates the patient to continue performing the rehabilitation sessions, and the automatic difficulty adjustment system, which allows the game to adjust to the patient's skills and avoid frustration. He also stated that this game could also be used in the treatment of other conditions that require hand and wrist rehabilitation, beyond stroke. The professional also highlighted the device chosen, which is, in his opinion, an easy and non-invasive tool to use, with 
no need of wires or complicated apparatus to work. As the main drawback, he stated that this system would be indicated for use only with patients which could perform movements against gravity.

Professional 3 expressed that he liked the game. As the main advantages, he indicated that the motivational aspect of the game is important. After a stroke, the patients become very dependent and the treatment is very passive, as there will be a professional helping them perform the exercises. With the game, they have a more active role in the treatment, improving their selfesteem and helping to reduce, in his opinion, the abandonment rates. He also enjoyed the ludic nature of the game and the device used, which he thought was simple, non-invasive and did not make the patients afraid to break it, while also having a relative low cost compared to other equipment commonly employed in rehabilitation systems. Regarding the game elements, he indicated that the game is simple, has an attractive story and the menus and visual feedback are clear.

\section{Discussion and Conclusion}

The final product of this work is a serious game for hand and wrist rehabilitation that incorporates all the main gestures and movements involved in this kind of rehabilitation. The game also tackles the main problems found in games for this purpose as discussed in previous sections: cost, patient motivation and adaptability.

The cost, usually related to the choice of high-cost devices, was addressed by using an offthe-shelf solution. However, it was necessary to design, test and integrate proper routines to deal with recognition of gestures not originally supported by the software of the chosen device. Another problem frequently found in the adoption of specific devices is related to handling complexity. It occurs when developers try to adapt a low-cost device to a system without considering user experience. By integrating the Myo, a natural interaction device, the adaptation time was decreased, and the user comfort increased. With the use of commercial devices of lower cost, the implementation of the game in rehabilitation clinics is easier.

To improve patient motivation, different approaches were used. An adaptive difficulty system was implemented, aiming to keep the game in a level of difficulty adequate to the player, avoiding frustrating the patient with exceedingly difficult tasks. An achievement system was also created, to provide them with goals and clear objectives that patients could aim for, further increasing player experience. Additionally, we applied the ideas from the expanded Schell's tetrad to improve patient experience with the game, integrating the four elements of the game together with the specific content, which in this case is hand and wrist rehabilitation.

An initial evaluation of the game was performed with rehabilitation professionals. It suggested that they believe the game could be important in the recovery process, highlighting especially the device, which they thought was simple, easy to use and had an accessible cost. They also stated that the system has potential to motivate patients to perform exercises and that they believe the automatic difficulty adjustment is important in this process. Regarding the game's interface, they indicated that it is clean and simple. This feedback suggests that the game is a valid option for assisting the treatment of patients and that its implementation in a clinic would be welcome. It also indicates that after being familiarized with the system, patients could take the system home to perform some exercises by themselves.

The contributions of this work are both technological and social. In the technological aspect, this work shows that it is possible to build rehabilitation systems with low-cost devices. The detection of new gestures that were previously not recognized by the device and the methodology involved can also be seen as contributions. In the social aspect, the development of a serious game that can be applied in a rehabilitation clinic or even at the patient's home, with the use of a lowcost device is a contribution to the recovery process of patients in rehabilitation treatment after a stroke or other debilitating condition. 
As future work, long term tests to evaluate patient commitment with the rehabilitation treatment could be performed. In this case, the element of interest is to observe if there is evidence of difference in withdrawal rates of groups that used the game during the rehabilitations sessions and groups that performed the traditional treatment.

From the technological point of view, we want to verify if the difference in the individual's electromyographic signals has an impact on the decision model's accuracy, possibly recruiting people to make a larger database to see if it improves the accuracy of the system. In addition, further research may be necessary to find different ways to process data and perform feature extraction, which may enhance the classification of gestures and improve game experience.

\section{Acknowledgements}

This project was supported by CAPES (process 1549360) and CNPq (processes 310561/2012-4 and 132170/2017-5).

\section{References}

[1] Global status report on noncommunicable diseases: 2014. World Health Organization, 2015.

[2] A. A. Blank, J. A. French, A. U. Pehlivan, and M. K. O'Malley, "Current Trends in Robot-Assisted Upper-Limb Stroke Rehabilitation: Promoting Patient Engagement in Therapy" Current Physical Medicine and Rehabilitation Reports, vol. 2, no. 3, pp. 184-195, September 2014. https://doi.org/10.1007/s40141-014-0056-z

[3] L. Brewer, F. Horgan, A. Hickey, and D. Williams, "Stroke rehabilitation: recent advances and future therapies," QJM, vol. 106, no. 1, pp. 11-25, January 2013. https://doi.org/10.1093/qjmed/hcs174

[4] M. Musicco, L. Emberti, G. Nappi, and C. Caltagirone, "Early and long-term outcome of rehabilitation in stroke patients: The role of patient characteristics, time of initiation, and duration of interventions" Archives of Physical Medicine and Rehabilitation, vol. 84, no. 4, pp. 551-558, April 2003. https://doi.org/10.1053/apmr.2003.50084

[5] B. Bonnechre, B. Jansen, L. Omelina, and S. Jan, "Do Patients Perform Their Exercises at Home and why (not)? A Survey on Patients' Habits during Rehabilitation Exercises" The Ulutas Medical Journal, vol. 2, no. 1, p. 41, 2016. DOI: 10.5455/umj.20160210060312

[6] N. Hocine, A. Gouaïch, and S. A. Cerri, "Dynamic Difficulty Adaptation in Serious Games for Motor Rehabilitation," in S. Göbel and J. Wiemeyer, (Eds.), Games for Training, Education, Health and Sports, vol. 8395, Cham: Springer International Publishing, 2014, pp. 115-128. https://doi.org/10.1007/978-3-319-05972-3_13

[7] E. Tsekleves, I. T. Paraskevopoulos, A. Warland, and C. Kilbride, "Development and preliminary evaluation of a novel low cost VR-based upper limb stroke rehabilitation platform using Wii technology" Disability and Rehabilitation: Assistive Technology, pp. 1-10, November 2014. https://doi.org/10.1007/978-3-319-05972-3_13

[8] I. Pastor, H. A. Hayes, and S. J. M. Bamberg, "A feasibility study of an upper limb rehabilitation system using kinect and computer games" in 2012 Annual International Conference of the IEEE Engineering in Medicine and Biology Society, San Diego, CA, 2012, pp. 1286-1289. DOI: 10.1109/EMBC.2012.6346173

[9] S. Sadeghi Esfahlani, B. Muresan, A. Sanaei, and G. Wilson, "Validity of the Kinect and Myo armband in a serious game for assessing upper limb movement" Entertainment Computing, vol. 27, pp. 150156, August 2018. https://doi.org/10.1016/j.entcom.2018.05.003

[10] M. Ghassemi, R. Ranganathan, A. Barry, K. Triandafilou, and D. Kamper, "Introduction of an EMGControlled Game to Facilitate Hand Rehabilitation After Stroke,” in J. Ibáñez, J. González-Vargas, J. M. Azorín, M. Akay, and J. L. Pons, (Eds.), Converging Clinical and Engineering Research on Neurorehabilitation II, vol. 15, Cham: Springer International Publishing, 2017, pp. 451-455. https://doi.org/10.1007/978-3-319-46669-9_75 
[11] J. W. Burke, M. McNeill, D. Charles, P. Morrow, J. Crosbie, and S. McDonough, "Serious Games for Upper Limb Rehabilitation Following Stroke," in 2009 Conference in Games and Virtual Worlds for Serious Applications, Coventry, UK, 2009, pp. 103-110. DOI: 10.1109/VS-GAMES.2009.17

[12] S. Saini, D. R. A. Rambli, S. Sulaiman, M. N. Zakaria, and S. R. Mohd Shukri, "A low-cost game framework for a home-based stroke rehabilitation system," in 2012 International Conference on Computer \& Information Science (ICCIS), Kuala Lumpur, Malaysia, 2012, pp. 55-60. DOI: 10.1109/ICCISci.2012.6297212

[13] N. Hocine, A. Gouaïch, S. A. Cerri, D. Mottet, J. Froger, and I. Laffont, "Adaptation in serious games for upper-limb rehabilitation: an approach to improve training outcomes," User Modeling and UserAdapted Interaction, vol. 25, no. 1, pp. 65-98, March 2015. https://doi.org/10.1007/s11257-015-91546

[14] J. Schell, The Art of Game Design: A Book of Lenses, Second Edition. A K Peters/CRC Press, 2014. https://doi.org/10.1201/b17723

[15] L. S. Machado, T. K. de L. Costa, and R. M. de Moraes, "Multidisciplinaridade e o desenvolvimento de serious games e simuladores para educação em saúde,” Revista Observatório, vol. 4, no. 4, pp. 149172, June 2018. https://doi.org/10.20873/uft.2447-4266.2018v4n4p149

[16] T. Paternostro-Sluga et al., "Reliability and validity of the Medical Research Council (MRC) scale and a modified scale for testing muscle strength in patients with radial palsy," Journal of Rehabilitation Medicine, vol. 40, no. 8, pp. 665-671, 2008. https://doi.org/10.2340/16501977-0235

[17] S. Mitra and T. Acharya, "Gesture Recognition: A Survey," IEEE Transactions on Systems, Man and Cybernetics, Part C (Applications and Reviews), vol. 37, no. 3, pp. 311-324, May 2007. https://doi.org/10.1109/TSMCC.2007.893280

[18] A.-A. Samadani and D. Kulic, "Hand gesture recognition based on surface electromyography," in 2014 36th Annual International Conference of the IEEE Engineering in Medicine and Biology Society, Chicago, IL, 2014, pp. 4196-4199. https://doi.org/10.1109/EMBC.2014.6944549

[19]R. Merletti and P. Parker, Eds., Electromyography: Physiology, Engineering, and Noninvasive Applications. Hoboken, NJ, USA: John Wiley \& Sons, Inc., 2004. DOI:10.1002/0471678384

[20] G. Kamen and D. A. Gabriel, Essentials of electromyography. Champaign, IL: Human Kinetics, 2010.

[21] S. Pizzolato, L. Tagliapietra, M. Cognolato, M. Reggiani, H. Müller, and M. Atzori, "Comparison of six electromyography acquisition setups on hand movement classification tasks," PLOS ONE, vol. 12, no. 10, p. e0186132, October 2017. https://doi.org/10.1371/journal.pone.0186132

[22]F. Kerber, M. Puhl, and A. Krüger, "User-independent real-time hand gesture recognition based on surface electromyography," in Proceedings of the 19th International Conference on Human-Computer Interaction with Mobile Devices and Services - MobileHCI '17, Vienna, Austria, 2017, pp. 1-7. https://doi.org/10.1145/3098279.3098553

[23] “LeapMotion”,https:/www.leapmotion.com/

[24]"Kai: The Gesture Controller for All Your Tech”, https://www.indiegogo.com/projects/kai-thegesture-controller-for-all-your-tech\#/

[25] R. Dörner, S. Göbel, W. Effelsberg, and J. Wiemeyer, Eds., Serious games: foundations, concepts and practice. Switzerland: Springer, 2016. DOI: 10.1007/978-3-319-40612-1

[26] P. Sweetser, D. Johnson, P. Wyeth, A. Anwar, Y. Meng, and A. Ozdowska, "GameFlow in Different Game Genres and Platforms," Computers in Entertainment, vol. 15, no. 3, pp. 1-24, Apr. 2017. https://doi.org/10.1145/3034780

[27]G. Alankus, A. Lazar, M. May, and C. Kelleher, "Towards customizable games for stroke rehabilitation," in Proceedings of the 28th international conference on Human factors in computing systems - CHI '10, Atlanta, Georgia, USA, 2010, p. 2113. https://doi.org/10.1145/1753326.1753649

[28] N. Hocine and A. Gouaich, “Therapeutic games' difficulty adaptation: An approach based on player's ability and motivation," in 2011 16th International Conference on Computer Games (CGAMES), Louisville, KY, USA, 2011, pp. 257-261. https://doi.org/10.1109/CGAMES.2011.6000349

[29]E. Criswell, Cram's introduction to surface electromyography. Jones \& Bartlett Publishers, 2010.

[30] S. Haykin, Neural networks: a comprehensive foundation. Prentice Hall PTR, 1994.

[31] T. V. V. Batista, R. M. Moraes, L. S. Machado, and A. M. G. Valenca, "Evaluating User Gestures in Rehabilitation from Electromyographic Signals" IEEE Latin America Transactions, vol. 14, no. 3, pp. 1387-1392, March 2016. https://doi.org/10.1109/TLA.2016.7459625

[32]N. Pentreath, Machine learning with Spark: create scalable machine learning applications to power a modern data-driven business using Spark. Birmingham: Packt Publ, 2015.

[33]F. Hayes-Roth, "Rule-based systems" Communications of the ACM, vol. 28, no. 9, pp. 921-932, September 1985. https://doi.org/10.1145/4284.4286 